\title{
O016: Electronic hand hygiene monitoring for the WHO 5-moments method
}

\author{
T Diller ${ }^{1 *}$, JW Kelly ${ }^{1}$, C Steed ${ }^{1}$, D Blackhurst ${ }^{1}$, S Boeker ${ }^{1}$, P Alper ${ }^{2}$ \\ From 2nd International Conference on Prevention and Infection Control (ICPIC 2013) \\ Geneva, Switzerland. 25-28 June 2013
}

\section{Introduction}

Two primary hand hygiene $(\mathrm{HH})$ methods exist. The In/ Out method teaches staff to clean their hands at entry to and exit from patient rooms. This method is easy to measure, but suffers from the potential for recontamination of staff from fomites after room entry. The World Health Organization (WHO) 5-Moments method teaches additional $\mathrm{HH}$ opportunities (HHOs) after entry to the patient room. It is difficult to measure and requires known direct observation. We describe the development and validation of a 5-Moments electronic monitoring system $\left(\right.$ DebMed $^{\mathbb{B}}$ GMS $\left.^{\mathrm{TM}}\right)$.

\section{Methods}

The (DebMed ${ }^{\circledR}$ GMS $\left.^{\mathrm{TM}}\right)$ captures soap/sanitizer dispenser activations with an implanted RF circuit board in the dispensers and transmits data via a wireless network to offsite servers. The activations represent the numerator. We previously developed an algorithm driven by patient census and nurse/patient staffing ratios to predict the number of HHOs (denominator) expected on medical/surgical, ICU or ED units (HOW2 Benchmark Study: AJIC 2011;39). The $\mathrm{HH}$ compliance index (HHCI) equals the activations divided by the predicted HHOs during a time period.

The HOW2 algorithm was derived from direct observation of periods of care activity, so we performed a validation study using 24 hour video-taped surveillance. We reviewed 1511 hours of video for 26 patients on a medical unit over 15 months and compared the actual $\mathrm{HH}$ compliance rate from the video-tape to the predicted HHCI from the (DebMed ${ }^{\circledR}$ GMS $\left.{ }^{\mathrm{TM}}\right)$.

\section{Results}

Quarterly HH compliance rates by direct observation ranged from 92 to $99 \%$. The electronic HHCI ranged from 65

'Greenville Health System, Greenville SC, USA to $71 \%$, while actual $\mathrm{HH}$ compliance from video-surveillance ranged from 66 to $75 \%$. Correlation of the latter two metrics was extremely high $(\mathrm{r}=0.976, \mathrm{p}=0.004)$. The number of HHOs with the In/Out method (2886) was $36 \%$ lower than with the 5-Moments method (4522).

\section{Conclusion}

This study validates the HOW2 Benchmark Study algorithm. It also documents a $36 \%$ deficit in HHOs using the In/Out method and a 30\% Hawthorne Effect due to direct observation. There is an extremely high correlation between actual video-taped $\mathrm{HH}$ compliance and the electronic monitoring system's HHCI. We believe that electronic monitoring using the 5-Moments method provides the most accurate and actionable $\mathrm{HH}$ compliance data.

\section{Disclosure of interest}

T. Diller Grant/Research support from Deb Worldwide Healthcare, Inc., J. Kelly Grant/Research support from Deb Worldwide Healthcare, Inc., C. Steed Grant/Research support from Deb Worldwide Healthcare, Inc., D. Blackhurst Grant/Research support from Deb Worldwide Healthcare, Inc., S. Boeker Grant/Research support from Deb Worldwide Healthcare, Inc., P. Alper Employee of Deb Worldwide Healthcare, Inc.

\section{Author details \\ ${ }^{1}$ Greenville Health System, Greenville SC, USA. ${ }^{2}$ Deb Worldwide Healthcare Inc., Charlotte NC, USA.}

Published: 20 June 2013

doi:10.1186/2047-2994-2-S1-016

Cite this article as: Diller et al:: O016: Electronic hand hygiene monitoring for the WHO 5-moments method. Antimicrobial Resistance and Infection Control 2013 2(Suppl 1):016.
Full list of author information is available at the end of the article

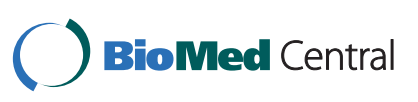

